\title{
Three-dimensional relativistic particle-in-cell hybrid code based on an exponential integrator
}

\author{
T. Tückmantel, A. Pukhov \\ Institut für Theoretische Physik I \\ Heinrich-Heine Universität Düsseldorf \\ 40225, Düsseldorf, Germany
}

\author{
J. Liljo \\ Mathematisches Institut \\ Heinrich-Heine Universität Düsseldorf \\ 40225, Düsseldorf, Germany
}

\author{
M. Hochbruck \\ Institut für Angewandte \\ und Numerische Mathematik \\ Karlsruher Institut für Technologie \\ 76128 Karlsruhe, Germany
}

\begin{abstract}
In this paper we present a new three dimensional (3D) full electromagnetic relativistic hybrid plasma code H-VLPL (hybrid virtual laser plasma laboratory). The full kinetic particlein-cell (PIC) method is used to simulate low density hot plasmas while the hydrodynamic model applies to the high density cold background plasma. To simulate the linear electromagnetic response of the high density plasma, we use a newly developed form of an exponential integrator method. It allows us to simulate plasmas of arbitrary densities using large time steps. The model reproduces the plasma dispersion and gives correct spatial scales like the plasma skin depth even for large grid cell sizes. We test the hybrid model validity by applying it to some physical examples.
\end{abstract}

\section{INTRODUCTION}

It is well accepted now that the particle-in-cell (PIC) codes provide the most detailed description of plasmas and are the key computational tools in the study of relativistic laser-plasma interactions [1], [2]. Large full 3D parallel electromagnetic simulation codes like VLPL [3], OSIRIS [4], VORPAL [5], OOPIC [6], and others contributed remarkably in our understanding of the complex laser-plasma physics. Because these codes provide the most detailed plasma description, they are computationally expensive. As a result one continues to look for new algorithms and simulation techniques to cope with challenges of the laser-plasma physics.

One of the reasons why the classical explicit PIC methods are computationally extremely expensive is that they have to resolve the fundamental plasma frequency $\omega_{p}=\sqrt{4 \pi n_{e} e^{2} / m_{e}}$, which is the frequency of the plasma electrostatic oscillations. Therefore, they are efficient only when applied to low density plasmas.

At the same time, there is a number of important applications where lasers interact with high density plasmas, e.g., the studies of electron propagation through solid density targets and the resulting target normal sheath acceleration (TNSA) [7]. The solid state density plasma densities are in the range of $100-1000 n_{c}$, where

$$
n_{c}=m \omega^{2} / 4 \pi e^{2}
$$

is the critical plasma density. Here, $m$ is the electron mass, $-e$ is its charge, and $\omega$ is the laser frequency. For the $1 \mu \mathrm{m}$ wavelength laser the critical electron density is $10^{21} 1 / \mathrm{cc}$. Other important applications include the Fast Ignition (FI) physics in the Inertial Confinement Fusion (ICF) studies [8]. The FI plasma has a density of the 1000 times compressed solid hydrogen, i.e., of the order of $10^{5} n_{c}$. Hence, the applicability of the classical PIC codes in this density range is facing severe difficulties. In this situation, one is forced to look for a more efficient numerical method to challenge those ultra-high densities. One of the possibilities is to include a hydrodynamic description of the high density plasma in the fully kinetic PIC code.

In last years PIC-hydrodynamic hybrid techniques have emerged as an efficient solution to large scale ultra highdensity plasma simulations, e.g., FI physics, solid state density plasma interactions, high charge, high energy ion generations etc [9]-[11]. Most of these codes work in the Darwin approximation and thus exclude the electromagnetic wave propagation completely. They also exclude electrostatic waves keeping the collisional magnetohydrodynamics (MHD) only. Further, an implicit electrostatic particle-fluid hybrid plasma code has been developed by Rambo and Denavit [12], which has been used to study interpenetration and ion separation in colliding plasmas [13]. There is also the implicit electromagnetic PIC code LSP [14]. This code uses an implicit global scheme which overcomes such restrictions of the time-step. The LSP code also employs a field solver based on an unconditionally Courant-stable algorithm [15] for electromagnetic calculations.

Recently, we have presented a 1D version of the code Hybrid Virtual Laser Plasma Laboratory (H-VLPL) [16] that unites a hydrodynamic model for overdense plasmas and the full kinetic description of hot low-density electrons and ions. In this code, the linear plasma response was simulated using an implicit scheme. The implementation involved the solution of linear systems, which have been done in a very efficient way using the Schur complement.

Unfortunately, the efficiency of the implicit H-VLPL code drops significantly if we extend the code from 1D to 3D. Therefore, we introduce a new 3D version of the code that is based on a different approach. Instead of using an implicit method, we employ a new variant of an exponential integrator [17] to model the high frequency plasma response. Exponential 
integrators are methods which make use of matrix functions related to the matrix exponential of the Jacobian of the differential equation. Here we construct a new method which is motivated by the mollified impulse method [18] proposed for molecular dynamics simulations.

The mollified impulse method is derived from a splitting approach. Variants of splitting methods are widely used for problems acting on different time scales, see [19]. For our hybrid model it turns out that due to the high density of the plasma, the highest frequencies stem from a multiplication operator, which acts only locally on each grid point. Frequencies arising from the Maxwellian part are much lower and can be handled explicitly as in the PIC code. This allows to implement the new mollified impulse method by evaluating matrix functions of diagonal matrices only. Obviously, this is much more efficient than the solution of linear systems resulting from a $3 \mathrm{D}$ discretization.

To illustrate the performance of the new method, we apply it to a few physical test examples. We check the correct dispersion of electromagnetic waves in the hybrid plasma and also compare the numerical skin length with the analytical expressions. As a more complicated test, we apply the new 3D code to the laser-solid interaction and acceleration of protons in the TNSA regime. Additionally, we perform a feasibility study of simulations of the Weibel instability, which occurs in the FI scenario.

The paper is organized as follows. First, we describe the full hybrid method in Section II. Then, we briefly explain the numerical scheme in Section III and finally, we test the new code H-VLPL on some well-known physical examples in Section IV.

\section{HYBRID MODEL}

In this section we define the physical model we use to simulate the plasma. We use the fully kinetic description with the usual PIC macroparticles for low density hot electrons and ions. The high density cold background plasma is then described hydrodynamically. Since the spatial locations of the kinetic particles and the hydrodynamic parts may overlap, we have to add currents generated by all species in the same grid cells. Note that, in contrast to usual approaches to problems acting on different time scales, we do not have separated spatial regions for the kinetic particles and the hydrodynamic part. The computation of the momentum of the hydrodynamically treated particles is combined with the electromagnetic field solver.
The equations for the fields and particle momenta read

$$
\begin{aligned}
\frac{\partial \mathbf{E}}{\partial t} & =c \nabla \times \mathbf{B}-4 \pi \sum_{\ell} \mathbf{j}_{\ell}, \quad \ell=e, i, h \\
\frac{\partial \mathbf{B}}{\partial t} & =-c \nabla \times \mathbf{E} \\
\frac{d \mathbf{p}_{h}}{d t} & =q_{e} \mathbf{E} \\
\frac{d \mathbf{p}_{\ell}}{d t} & =q_{\ell}\left(\mathbf{E}+\frac{\mathbf{v}_{\ell}}{c} \times \mathbf{B}\right), \quad \ell=e, i
\end{aligned}
$$

where

$$
\mathbf{j}_{\ell}=q_{\ell} n_{\ell} \mathbf{v}_{\ell}, \quad \mathbf{p}_{\ell}=m_{\ell} \gamma_{\ell} \mathbf{v}_{\ell}, \quad \gamma_{\ell}=\sqrt{1+\frac{\mathbf{p}_{\ell}^{2}}{\left(m_{\ell} c\right)^{2}}}
$$

The index $\ell=e, i, h$ denotes electrons, ions, and hybrid particles, respectively. $\mathbf{E}$ and $\mathbf{B}$ denote the electric and magnetic field vectors, $\mathbf{j}$ denotes the current density, $\mathbf{p}$ is the momentum and $n$ the number density of particles.

In the momentum equation (2c) we have neglected the nonlinear part of the Lorentz force $\mathbf{v} \times \mathbf{B} / c$, because we assume that the velocity of the electron part in the cold background plasma is small, $v \ll c$. This assumption, however, limits the cold plasma response to the linear one.

\section{NUMERICAL ALGORITHM}

For simplicity, we rewrite the equations in dimensionless variables, $\widetilde{t}=\omega_{0} t$ and $\widetilde{x}=k_{0} x$, where $\omega_{0}$ denotes the laser frequency and $k_{0}=\omega_{0} / c$. The new variables are then

$$
\widetilde{E}=\frac{e E}{m_{e} c \omega_{0}}, \quad \widetilde{B}=\frac{e B}{m_{e} c \omega_{0}}, \quad \widetilde{p}_{h}=\frac{p_{h}}{m_{e} c}, \quad \widetilde{p}_{\ell}=\frac{p_{\ell}}{m_{\ell} c},
$$

and

$$
\widetilde{j}_{\ell}=\frac{j_{\ell}}{j_{c}}, \quad j_{c}=e n_{c} c, \quad \widetilde{n}=\frac{n}{n_{c}}, \quad \widetilde{q}_{\ell}=\frac{q_{\ell} m_{e}}{e m_{\ell}}, \quad \widetilde{v}_{\ell}=\frac{v_{\ell}}{c},
$$

where $\ell=e, i$.

In the following, we omit the tildes, neglect the Lorentz force and consider hybrid particles only. We can then write $\mathbf{p}=\mathbf{p}_{h}$. Using these simplification, Eq. (2) reads

$$
\begin{aligned}
& \frac{\partial \mathbf{E}}{\partial t}=\nabla \times \mathbf{B}+\omega_{p}^{2} \mathbf{p} \\
& \frac{\partial \mathbf{B}}{\partial t}=-\nabla \times \mathbf{E} \\
& \frac{d \mathbf{p}}{d t}=-\mathbf{E},
\end{aligned}
$$

where $\omega_{p}^{2}=\frac{n_{h}}{\gamma_{h}}$.

The problem is considered in three space dimensions. We solve the equations on a staggered grid and approximate the spatial derivatives with centered finite differences using the Yee scheme [20]. 
For the time discretization we will use the following splitting of the vector fields

$$
\begin{aligned}
{\left[\begin{array}{c}
\dot{\mathbf{p}} \\
\dot{\mathbf{E}} \\
\dot{\mathbf{B}}
\end{array}\right] } & =\left[\begin{array}{c}
-\mathbf{E} \\
\nabla \times \mathbf{B}+\omega_{p}^{2} \mathbf{p} \\
-\nabla \times \mathbf{E}
\end{array}\right] \\
& =\left[\begin{array}{c}
0 \\
0 \\
-\nabla \times \mathbf{E}
\end{array}\right]+\left[\begin{array}{c}
0 \\
\nabla \times \mathbf{B} \\
0
\end{array}\right]+\left[\begin{array}{c}
-\mathbf{E} \\
\omega_{p}^{2} \mathbf{p} \\
0
\end{array}\right]
\end{aligned}
$$

If $\omega_{p}$ is constant over a time step, the exact solution of each of the three differential equations

$$
\left[\begin{array}{c}
\dot{\mathbf{p}} \\
\dot{\mathbf{E}} \\
\dot{\mathbf{B}}
\end{array}\right]=\left[\begin{array}{c}
0 \\
0 \\
-\nabla \times \mathbf{E}
\end{array}\right], \quad\left[\begin{array}{c}
\dot{\mathbf{p}} \\
\dot{\mathbf{E}} \\
\dot{\mathbf{B}}
\end{array}\right]=\left[\begin{array}{c}
0 \\
\nabla \times \mathbf{B} \\
0
\end{array}\right], \quad\left[\begin{array}{c}
\dot{\mathbf{p}} \\
\dot{\mathbf{E}} \\
\dot{\mathbf{B}}
\end{array}\right]=\left[\begin{array}{c}
-\mathbf{E} \\
\omega_{p}^{2} \mathbf{p} \\
0
\end{array}\right]
$$

can be computed very efficiently, in particular without solving any linear system. A symmetric splitting yields the following scheme

$$
\begin{aligned}
B^{n+\frac{1}{2}} & =B^{n}-\frac{\tau}{2} \nabla \times E^{n} \\
\left(E^{+}\right)^{n} & =E^{n}+\frac{\tau}{2} \nabla \times B^{n+\frac{1}{2}} \\
{\left[\begin{array}{c}
p^{n+1} \\
\left(E^{-}\right)^{n+1}
\end{array}\right] } & =\left[\begin{array}{cc}
\cos \tau \omega_{p}^{n} & \tau \operatorname{sinc} \tau \omega_{p}^{n} \\
-\omega_{p}^{n} \sin \tau \omega_{p}^{n} & \cos \tau \omega_{p}^{n}
\end{array}\right]\left[\begin{array}{c}
p^{n} \\
\left(E^{+}\right)^{n}
\end{array}\right] \\
E^{n+1} & =\left(E^{-}\right)^{n+1}+\frac{\tau}{2} \nabla \times B^{n+\frac{1}{2}} \\
B^{n+1} & =B^{n+\frac{1}{2}}-\frac{\tau}{2} \nabla \times E^{n+1} .
\end{aligned}
$$

Although this splitting method is of classical order two (since it is a symmetric scheme), it suffers from resonances, which arise in $E, B$, and $p$ if the density becomes large. In fact, the errors of this scheme are of order zero for certain time steps. We illustrate this effect by simulating a 1D plane wave. The incoming laser pulse is modeled via inhomogeneous, time dependent Dirichlet boundary conditions and zero as initial values. A spatial grid size of 0.5 for $x \in[0,200]$ is used. The hybrid density is set to $n_{h}=10^{8} n_{c}$ and the system is integrated over the time interval $[0,200]$. The blue curves in Fig. 1 shows the errors in $E_{y}, B_{z}$ and $p_{y}$ of the splitting method (5) as a function of the time step size, while the red line corresponds to a second-order error behavior. To improve the presentation, we only show the interval $[0.25,0.5]$ for the time steps, but we would like to emphasize that the same effects have been obtained for much smaller time steps as well.
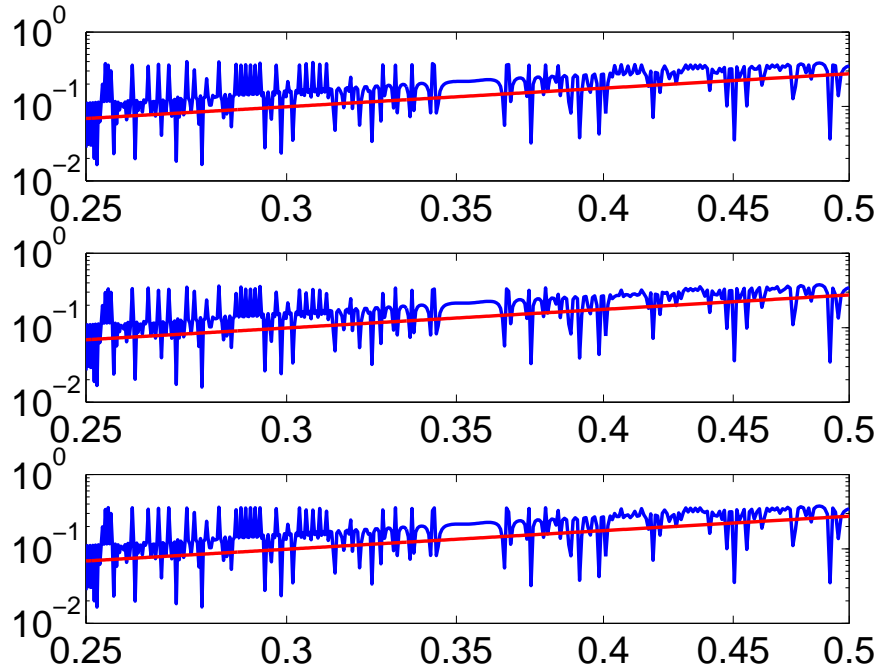

Fig. 1. Error in $E_{y}, B_{z}$ and $p_{y}$ plotted over the step size (blue) for a straightforward integration of Eq. (4). The red line shows the expected order two.

Similar resonance effects have also been observed for multiple time stepping schemes in molecular dynamics simulations [21] and for numerical methods for solving second-order differential equations [18], [22], [23]. Motivated by these papers, we suggest to apply filter functions and averaging operators to the Maxwellian part and modify the splitting method (5) in the following way

$$
\begin{aligned}
B^{n+\frac{1}{2}} & =B^{n}-\frac{\tau}{2} \nabla \times \phi\left(\tau \omega_{p}^{n}\right) E^{n} \\
\left(E^{+}\right)^{n} & =E^{n}+\frac{\tau}{2} \psi\left(\tau \omega_{p}^{n}\right) \nabla \times B^{n+\frac{1}{2}} \\
{\left[\begin{array}{c}
p^{n+1} \\
\left(E^{-}\right)^{n+1}
\end{array}\right] } & =\left[\begin{array}{cc}
\cos \tau \omega_{p}^{n} & \tau \operatorname{sinc} \tau \omega_{p}^{n} \\
-\omega_{p}^{n} \sin \tau \omega_{p}^{n} & \cos \tau \omega_{p}^{n}
\end{array}\right]\left[\begin{array}{c}
p^{n} \\
\left(E^{+}\right)^{n}
\end{array}\right] \\
E^{n+1} & =\left(E^{-}\right)^{n+1}+\frac{\tau}{2} \psi\left(\tau \omega_{p}^{n}\right) \nabla \times B^{n+\frac{1}{2}} \\
B^{n+1} & =B^{n+\frac{1}{2}}-\frac{\tau}{2} \nabla \times \phi\left(\tau \omega_{p}^{n}\right) E^{n+1} .
\end{aligned}
$$

Clearly, a detailed analysis is necessary to fully understand how to choose the filter function $\phi$ and $\psi$ such that second order error estimates or long time energy conservation can be guaranteed under meaningful assumptions on the solution. The situation here is much more complicated than the case of second order differential equations analyzed in [18], [22], [23]. The theoretical properties of the numerical method including a detailed error analysis are currently investigated and will be reported elsewhere. However, a preliminary analysis of local errors led us to try to use the filter functions $\phi(x)=\psi(x)=\operatorname{sinc}(x / 2)$, where $\operatorname{sinc}(x):=\sin (x) / x$ and to verify these properties numerically. Fig. 2 shows the same numerical test as for Fig. 1 with these filter functions and time steps $\tau \in[0.1,0.5]$. The resonances have been eliminated completely and order two is achieved for arbitrarily large densities. 

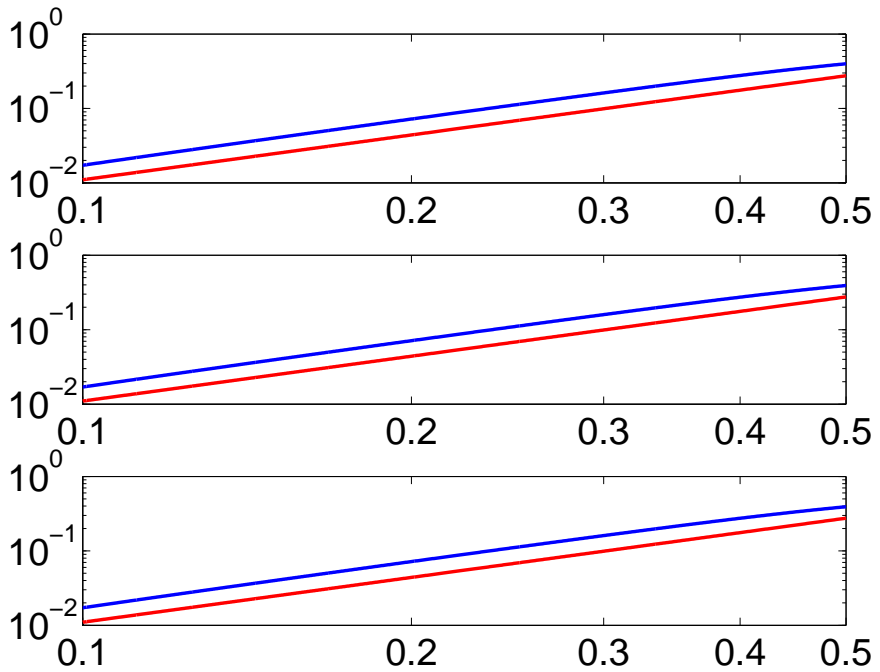

Fig. 2. Error in $E_{y}, B_{z}$ and $p_{y}$ plotted over the step size (blue) with $\phi(x)=\psi(x)=\operatorname{sinc} \frac{x}{2}$. The red line indicates the expected order two.

\section{BENCHMARK WITH PHYSICAL PROCESSES}

The numerical integrator described in the previous sections has been implemented into the VLPL code as a three-dimensional, parallelized version, and is now operational. In order to examine its accuracy and reliability, we have benchmarked it with a variety of physical processes.

First, we check if it correctly models laser propagation through linearly dispersive plasma as well as reflection from overdense plasma. Second, we verify the conservation of the total energy of the system by the hybrid algorithm. Third, our code is applied to the Target Normal Sheath Acceleration (TNSA) process, which would have been very difficult to treat just using a direct PIC code because of the high plasma frequency at the solid state density. We check if our hybrid integrator correctly describes the exponential decay of a wave in overdense plasma. Finally, we show its applicability to study the Weibel instability.

\section{A. Reflection of an incident pulse}

As the simplest test one can imagine, we will show that our integrator accurately models refraction in underdense plasma and reflection from overdense plasma. First, we set up a plasma slab of $0.85 n_{c}$ density (1) and send a $26 f s$ Gaussian laser pulse through it. As the pulse hits the surface of the purely hybrid plasma, a part of the wave is transmitted while a significant reflection also occurs.
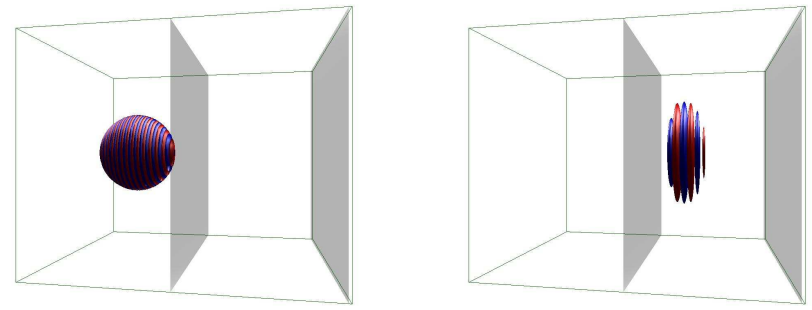

Fig. 3. Snapshots of the simulation setup taken with an interactive VR visualization software, which is a part of H-VLPL and currently under development. The left picture shows the laser pulse (isosurfaces of fixed positive and negative electric field amplitudes) as it enters the hybrid plasma. The right picture demonstrates the dispersive effect.

On the other hand, when the experiment was modified by setting the density to $1.2 n_{c}$, we observe a reflection of the entire electromagnetic wave by the plasma.

We point out that these simulations have been performed using just the fluid part of our combined code without any PIC macroparticles. Still, the effect has been described correctly.

\section{B. Energy conservation}

An important property we require from the new integrator is the conservation of the total energy of the system, comprising PIC macroparticles, electromagnetic fields, and the hybrid fluid. A very simple setup with a laser pulse being reflected from an overdense surface is used for this benchmark. We expect the total energy

$$
\begin{aligned}
E_{t o t}=\sum_{l} m_{l} c^{2}(\gamma-1) & +\frac{1}{8 \pi} \int_{V}\left(E^{2}+B^{2}\right) d V \\
& +\int_{V} n_{h}\left(\gamma_{h}-1\right) m_{h} c^{2} d V
\end{aligned}
$$

to be constant, where $m_{l}$ are the masses of the respective particle species and $\gamma=\sqrt{1+\left(p_{l} / m_{l} c\right)^{2}}$ is the relativistic gamma factor. We denote the hybrid density by $n_{h}$ and its gamma factor by $\gamma_{h}$. Figure 4 shows the total energy of the simulation versus time, which is measured in units of laser periods.

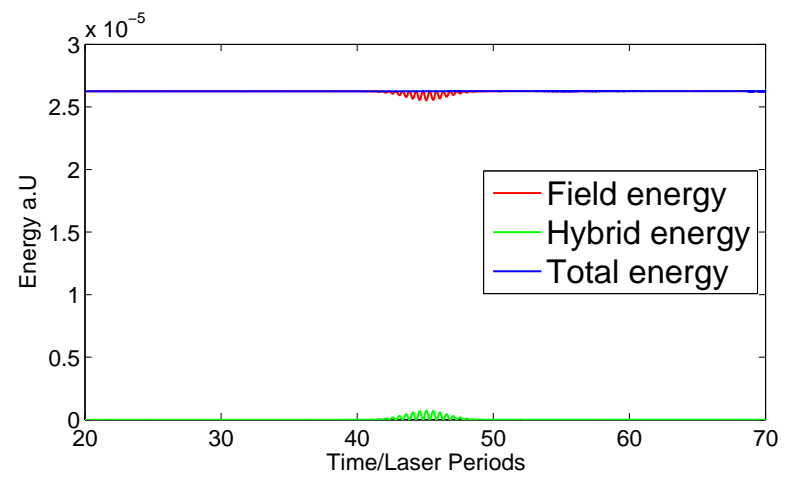

Fig. 4. Plot of the total energy, the energy of the electromagnetic field and of the hybrid plasma versus time. 
During the laser propagation in vacuum, the energy stays constant except for small fluctuations within the order of magnitude of the machine precision. When the pulse hits the overdense hybrid plasma surface, it is reflected, as can be seen at the time of 45 laser periods. While this reflection occurs, energy fluctuations are limited by $0.04 \%$ of the total energy.
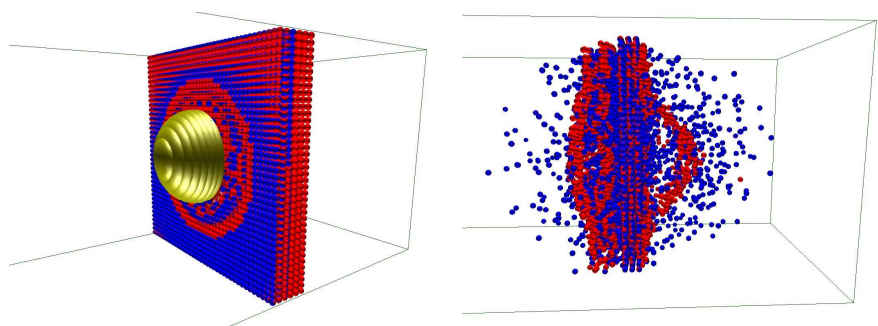

Fig. 6. Snapshot of the TNSA benchmark simulation after 10 (left) and 380 (right) laser periods. PIC macroparticles containing electrons are displayed blue, while those with protons are rendered red. One observes the thin coating of protons dissolving from the back of the foil in the right image.

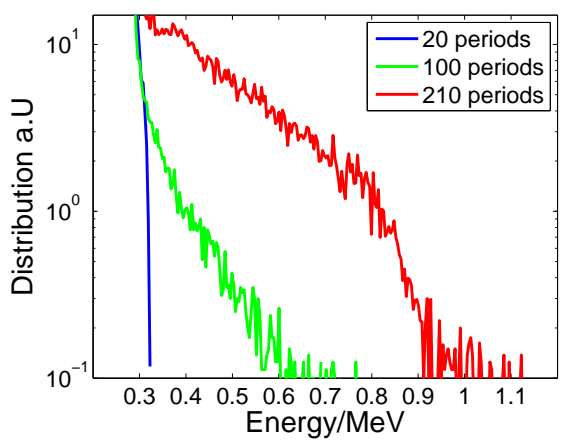

Fig. 7. Spectrum of the accelerated ions in the TNSA simulation. laser. The foil consists of a bulk part of $1000 n_{c}$, a preplasma on its front surface, and an $80 \mathrm{~nm}$ thick proton layer on its back surface. The preplasma is modeled as a density ramp reaching from 0 to $2 n_{c}$ over a distance of 2 laser wavelengths $(1.6 \mu \mathrm{m})$ and treated entirely by the PIC method. Analogously, we use PIC macroparticles for the back surface protons. On the contrary, any attempt to describe the highly overdense main part of the foil as macroparticles would result in numerical problems. Here we use the hydrodynamic feature of H-VLPL, setting the hybrid density on the grid to $1000 n_{c}$.

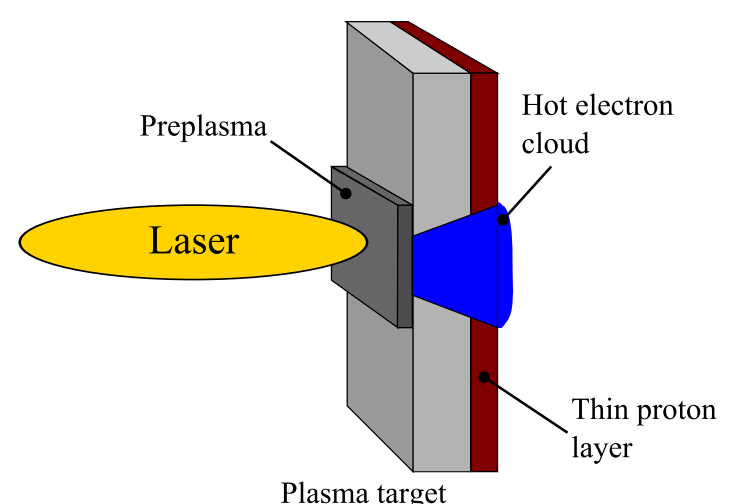

Fig. 5. Schematic of the TNSA process.
The intense laser radiation creates a blow-off region in the front of the foil, resulting in a large cloud of hot electrons, which, in parts, propagates through the foil and passes the coating of the back surface. As the electrons leave the surface, a strong electrostatic field is built up, and the protons are pulled out of the foil and eventually accelerated to high energies.

In Fig. 7, the spectrum of the accelerated ions is shown. A maximum energy of about $0.9 \mathrm{MeV}$ is reached, which is quite remarkable considering the laser intensity in the setup.

We conclude that our hybrid algorithm succeeded well and efficiently in treating this numerically challenging physical situation.

\section{Comparison of skin depths}

As a further benchmark for our hybrid code we check the decay of a wave in an overdense plasma. According to the linear theory, it should scale as $E(x) \sim \exp \left(-x / \delta_{s}\right)$ in the plasma, where $\delta_{s}=c / \sqrt{\omega_{p}^{2}-\omega^{2}}$ is the skin length. Several simulations have been set up using a circularly polarized laser pulse with duration $6 \lambda$ and amplitude $a_{0}=0.01$ in order to avoid relativistic nonlinearities. The densities of the plasma surfaces used for this benchmark range from $1.5 n_{c}$ to $500 n_{c}$. We show the decay of the wave inside the plasma for three densities; the agreement with the theoretical predictions up to densities of $500 n_{c}$ is very good. 
Additionally, by fitting exponentials through the measured field data, one can compute the skin depths of the decay. In figure 9, the results are shown and we get an excellent agreement. One has to mention that even though these simulations have been done with a grid step of $0.05 \lambda$, the skin depths match remarkably well with the theory up to a density of $500 n_{c}$, where $\delta_{s}=0.007 \lambda$.

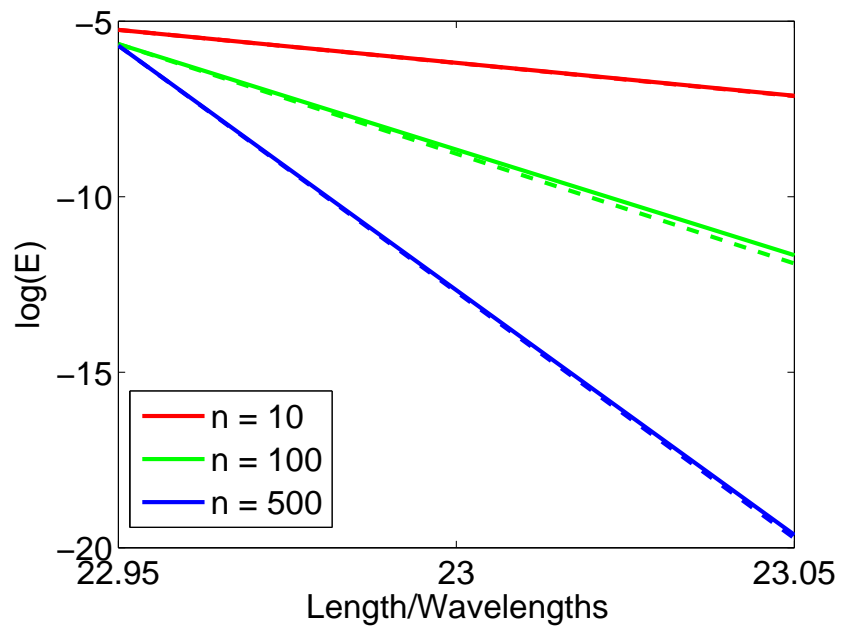

Fig. 8. Snapshot of the logarithm of the fields $\log \left(\sqrt{E_{y}^{2}+E_{z}^{2}}\right)$ (solid lines) inside the plasma for three different densities. The dashed lines show the theoretical prediction.

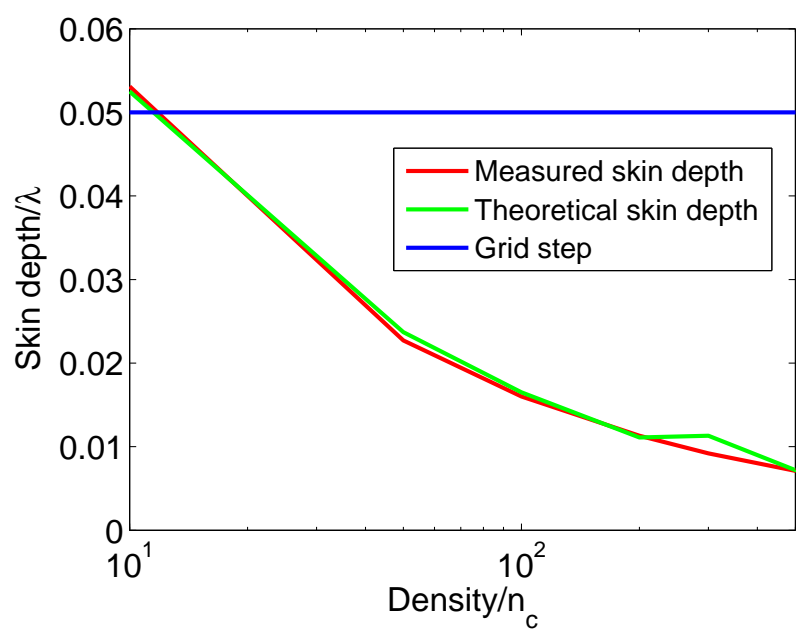

Fig. 9. Plot of the skin depth versus the plasma density. The blue line shows the grid step used in the simulation.

\section{E. Investigation of Weibel instabilities}

When studying the fast ignition (FI) scenario [8] in inertial confinement fusion, one is interested in the behavior of the beam of electrons propagating into the target, particularly the amount of energy deposited and the shaping of the beam over time. Generally, electron beams running through a background plasma suffer from the major problem of the Weibel instability [25], which is a very important issue to be studied if one wants to understand the FI scheme. The ratio of the beam density to that of the background $n_{b} / n_{p}$, as well as the density gradient in propagation direction, is likely to influence the evolution of the beam, its filamentation and the increase of electromagnetic fields as the instability builds up.

For low densities, roughly about $100 n_{c}$, PIC simulations can be carried out to perform these investigations. However, as the electron beam approaches the core of an ICF pellet, the density will exceed multiple times solid density and conventional PIC codes must be applied with extremely small time steps in order to avoid numerical instability, and thus cannot be used with reasonable computational effort.

We are going to study the phenomenon of the Weibel instability with our new hybrid code, using standard PIC macroparticles for the electron beam and the fluid part in order to model the background plasma. Since H-VLPL has no restrictions for the hybrid densities used, we can perform such simulations within a moderate amount of CPU time.

In order to obtain a proof for the physical correctness of our code within the linear regime, we have launched tests with H-VLPL comparing a classical PIC computation to a hybrid simulation of this setup. An electron beam with density $n_{b}$ propagates through a background plasma with $n_{p}=100 n_{b}$. The momentum of the beam electrons is $p_{b}=m c$ with a thermal spread of $10^{-4} \mathrm{mc}$, and the momentum of the background is chosen such that its current compensates for that of the beam plasma, meaning

$$
n_{b} v_{b}+n_{p} v_{p}=0
$$

The setup is restricted to a $2 \mathrm{D}$ geometry, with the beams traveling perpendicularly to the $x-y$-plane; this is necessary in order to exclude two-stream instabilities. After about 3.3 beam plasma periods $2 \pi / \omega_{b}$, with $\omega=\sqrt{4 \pi n_{b} e^{2} / m}$, one observes a strong filamentation of the beam, and a magnetic field builds up. When launching the same simulation with and without the hybrid model, we notice that the latter succeeds well in describing the filamentation effect at the initial, linear stage. We compare the integral of the squared magnetic field

$$
\int_{V} \mathbf{B}^{2} d V
$$

of the two models. At this point it has to be mentioned that during the nonlinear stage of the instability, the present version of H-VLPL will fail in describing the filamentation of the background plasma since it does not treat its continuity equation and convective term of momentum evolution. Additionally, the fluid plasma does not react to magnetic fields directly. 

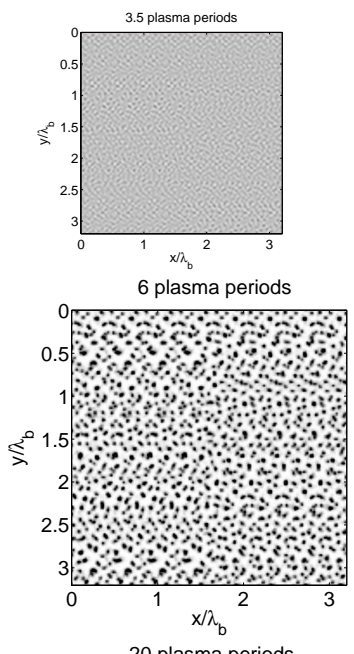

20 plasma periods

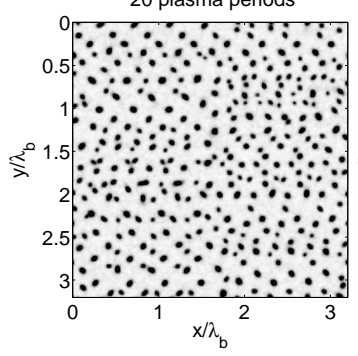

Fig. 10. Snapshot of the Weibel instability benchmark simulations with PIC (left) and the hybrid code (right) at 3.5, 6, and 20 beam plasma periods. We observe very similar behavior, although the instability starts approximately 0.5 period later with the hybrid model due to the lower numerical noise.

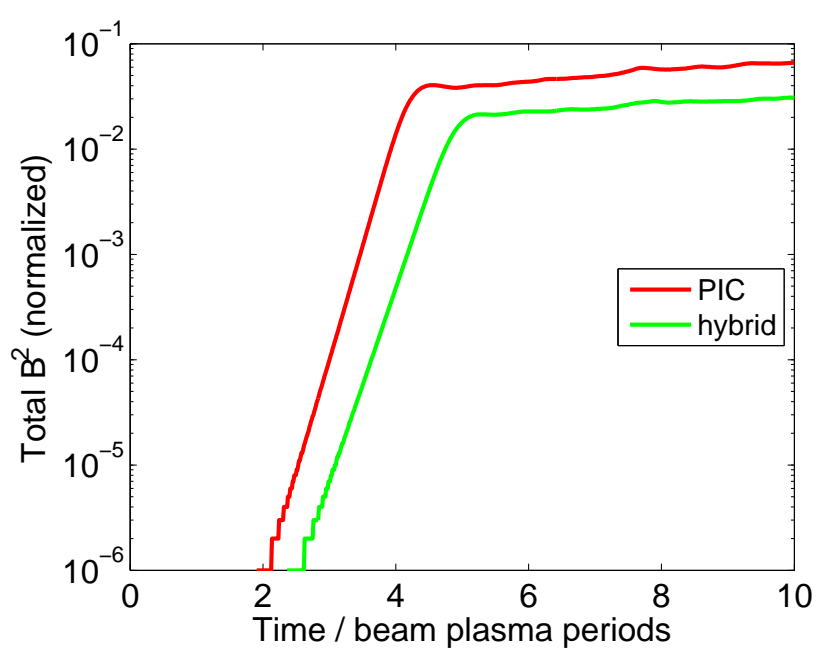

Fig. 11. Integral of the squared B-field over the simulation plane. During the linear stage of the instability, we observe an exponential growth with almost the same growth rate.

Nevertheless, the behavior, and the growth rate of the Weibel instability during the linear stage are accurately reproduced. This result indicates the applicability of H-VLPL to the Weibel instability scenario, and makes further investigations of the effect with an advanced, fully hydrodynamic hybrid code appear promising.

\section{OUTLOOK}

The next step in the development of our hybrid laser plasma simulation system H-VLPL will be the full nonlinear hydrodynamic description of the background plasma. This includes the continuity equation to describe the fluid transport as well as momentum transport equation. We are going to further study the physical effects mentioned above, namely the TNSA process and the Weibel instability, using an advanced version of H-VLPL, which is currently under development.

\section{ACKNOWLEDGEMENT}

This work has been supported by the Deutsche Forschungsgemeinschaft via GRK 1203 and SFB TR 18.

\section{REFERENCES}

[1] C. K. Birdsall, A. B. Langdon, "Plasma Physics Via Computer Simulation", Adam Higer, (1991).

[2] R. W. Hockney, J. W. Eastwood, Computer Simulation Using Particles, McGraw-Hill, London, (1981).

[3] Pukhov A., "Three-dimensional electromagnetic relativistic particle-incell code VLPL (Virtual Laser Plasma Lab)", J. Plasma Phys., vol. 61, pp. 425-433, Apr. 1999.

[4] R. A. Fonseca, L. O. Silva, F. S. Tsung, V. K. Decyk, W. Lu, C. Ren, W. B. Mori, S. Deng, S. Lee, T. Katsouleas, J. C. Adam, "OSIRIS: A threedimensional, fully relativistic particle in cell code for modeling plasma based accelerators", Lecture Notes in Computer Science, vol. 2331, pp. 342-351, 2002.

[5] C. Nieter, J. R. Cary, "VORPAL: a versatile plasma simulation code", $J$. Comput. Phys., vol. 196, pp. 448-473, May 2004.

[6] J. P. Verboncoeur, A. B. Langdon, N. T. Gladd, "An object-oriented electromagnetic PIC code", Comput. Phys. Commun., vol. 87(1-2), pp. 199-211, May 1995

[7] L. Robson, P. T. Simpson, R. J. Clarke, K. W. D. Ledingham, F. Lindau, O. Lundh, T. McCanny, P. Mora, D. Neely, C.-G. Wahlström, M. Zepf and P. McKenna, "Scaling of proton acceleration driven by petawatt-laserplasma interactions", Nature Physics, vol. bf 3(1), pp. 58-62, Jan. 2007. M. Roth, et al., "Energetic ions generated by laser pulses: A detailed study on target properties", Phys. Rev. Special Topics-Accelerators and Beams, vol. 5(6), Jun. 2002.

M. Kaluza et al., "Influence of the laser prepulse on proton acceleration in this-foil experiments", Phys. Rev. Lett. vol. 93, 045003, Jul. 2004.

[8] M. Tabak, J. H. Hammer, M. E. Glinsky, W. L. Kruer, S. C. Wilks, J. Woodworth, E. M. Campbell, M. D. Perry, and R. J. Mason, "Ignition and high-gain with ultrapowerful lasers", Phys. Plasmas, vol. 1, pp. 16261634, May. 1994.

[9] R. J. Mason, "Monte-Carlo hybrid modeling of electron-transport in laserproduced plasmas", Phys. Fluids, vol. 23(11), pp. 2204-2215, 1980.

[10] J. R. Davies, A. R. Bell, M. G. Haines, and S. Guerin, "Short pulse highintensity laser-generated fast electron transport into thick solid targets", Phys. Rev. E, vol. 56(6), pp. 7193-7203, Dec. 1997.

[11] L. Gremillet, G. Bonnaud, F. Amiranoff, "Filamented transport of lasergenerated relativistic electrons penetrating a solid target", Phys. Plasmas, vol. 9(3), pp. 941-948, Mar. 2002.

[12] P. W. Rambo and J. Denavit, "Fluid and field algorithms for time-implicit plasma simulation", J. Comp. Phys., vol. 92, pp. 185-212, Jan. 1991 (1991) 185.

[13] P. W. Rambo and J. Denavit, "Interpenetration and ion separation in colliding plasmas", Phys. Plasmas, vol. 1(12), pp. 4050-4060, Dec. 1994.

[14] D. R. Welch, D. V. Rose, R. E. Clark, T. C. Genoni, T. P. Hughes, "Implementation of a non-iterative implicit electromagnetic field solver for dense plasma simulation", Comput. Phys. Commun., vol. 164(1-3), pp. 183-188, Dec. 2004.

[15] F. Zheng, Z. Chen, IEEE Trans. Microwave Theory Tech. 49 (2001) 1006. 
[16] J. Liljo, A. Karmakar, A. Pukhov, M. Hochbruck, "One-dimensional electromagnetic relativistic PIC-hydrodynamic hybrid simulation code $\mathrm{H}$ VLPL (hybrid virtual laser plasma lab)", Comput. Phys. Commun., vol. 179(6), pp. 371-379, Sep. 2008.

[17] M. Hochbruck and A. Ostermann, "Exponential integrators", Acta Numerica, 2010, to appear

[18] B. García-Archilla, J. M. Sanz-Serna and R. D. Skeel (1998), "Longtime-step methods for oscillatory differential equations", SIAM J. Sci. Comput., vol. 20(3), pp. 930-963, Oct. 1998.

[19] E. Hairer, C. Lubich and G. Wanner (2006), "Geometric Numerical Integration, Structure-Preserving Algorithms for Ordinary Differential Equations", Springer Series in Computational Mathematics, vol. 31, Springer, Berlin, Heidelberg.

[20] K.S. Yee, "Numerical solution of initial boundary value problems involving Maxwell's equations in isotropic media", IEEE Trans. Antennas and Propagation, vol. AP14(3), pp. 302-307, 1966.

[21] J. J. Biesiadecki and R. D. Skeel, "Dangers of multiple time step methods", J. Comput. Phys., vol. 109(2), pp. 318-328, Dec. 1993.

[22] M. Hochbruck and C. Lubich, "A Gautschi-type method for oscillatory second-order differential equations", Numer. Math., vol. 83(3), pp. 403426, Sep. 1999.

[23] V. Grimm and M. Hochbruck (2006), "Error analysis of exponential integrators for oscillatory second-order differential equations", J. Phys. A, vol. 39(19), pp. 5495-5507, May. 2006

[24] S.C. Wilks, A.B. Langdon, T.E. Cowan, M. Roth, M. Singh, S. Hatchett, et. al., "Energetic proton generation in ultra-intense laser-solid interactions", Phys. Plasmas, vol. 8(2), pp. 542-549, Feb. 2001.

[25] E.S. Weibel, "Spontaneously growing transverse waves in a plasma due to an anisotropic velocity distribution", Phys. Rev. Lett., vol. 2(3), pp. 8384, 1959. 